\title{
EDUCACIÓN A DISTANCIA DEL PROFESORADO DE CIENCIAS EN EL DESARROLLO DE LABORATORIOS VIRTUALES
}

\author{
(DISTANCE EDUCATION ON VIRTUAL-LAB IMPLEMENTATION FOR SCIENCE TEACHERS)
}

Carla Martín Villalba

Alfonso Urquía Moraleda

Sebastián Dormido Bencomo

Universidad Nacional de Educación a Distancia, UNED (España)

\section{RESUMEN}

Se presentan dos cursos a distancia, sobre el desarrollo de laboratorios virtuales interactivos, que están especialmente dirigidos a profesores de materias de Ciencias que deseen aplicar eficazmente la simulación interactiva por ordenador en sus clases. El primero de ellos, "Laboratorios virtuales interactivos: un enfoque creativo para la enseñanza de las Ciencias en el aula y a través de Internet", pertenece al Programa de Formación del Profesorado de la UNED. El segundo curso, "Virtual-lab implementation with Ejs", es una versión online gratuita del anterior y está escrito en lengua inglesa. Este curso ganó el segundo premio del concurso "Best Designed Course Award", celebrado en 2005 con motivo de la $6^{\text {th }}$ International Conference on Virtual University (Bratislava, Eslovaquia). El entorno de simulación empleado en ambos cursos es Easy Java Simulations (Ejs): una herramienta gratuita, de código abierto, especialmente concebida para ser usada por personas con escasos conocimientos de programación.

El sitio web del curso gratuito online es http://www.euclides.dia.uned.es/simulab-pfp

Palabras clave: educación a distancia, educación basada en web, formación del profesorado, laboratorio virtual, simulación interactiva, Easy Java Simulations.

\begin{abstract}
Two distance courses on the implementation of interactive virtual-labs are presented in this manuscript. Their audience is Science teachers who wish to make efficient use of interactive simulation in their classes. The first course, entitled "Laboratorios virtuales interactivos: un enfoque creativo para la enseñanza de las Ciencias en el aula y a través de Internet”, is part of the Continuing Education Program offered by UNED, the National Open University of Spain. The second course is a concise version of the
\end{abstract}


first one. It is a free online course, written in English language, entitled "Virtual-lab implementation with Ejs". This web-based course got the second award in the "Best Designed Course" competition of the $6^{\text {th }}$ International Conference on Virtual University (December 2005, Bratislava, Slovak Republic). The simulation environment used to implement the virtual-labs is Easy Java Simulations (Ejs): a freeware, open source, Java-based tool conceived to be used by people with little programming skills.

Online course website: http://www.euclides.dia.uned.es/simulab-pfp

Keywords: distance education, web-based education, teacher training, virtual laboratory, interactive simulation, Easy Java Simulations.

Los laboratorios virtuales pueden ser herramientas docentes de gran utilidad, adecuados para la educación a distancia (Jimoyiannis y Komis, 2001). Son aplicaciones informáticas interactivas que simulan la evolución temporal de un modelo matemático y proporcionan información visual acerca de dicha evolución empleando gráficas, diagramas animados, etc. El laboratorio virtual se compone de un modelo y de una vista. Esta arquitectura modelo-vista es una simplificación de la arquitectura modelo-vista-control (Burbeck, 1997), ampliamente seguida en el diseño y programación de aplicaciones informáticas que tienen interfaces gráficas de usuario.

El modelo del laboratorio virtual es el modelo matemático y la vista es la información visual sobre el modelo. Además, la vista constituye la interfaz entre la simulación del modelo matemático y el usuario. La vista del laboratorio no sólo refleja de manera dinámica la evolución del modelo, sino que además permite al usuario modificar los parámetros y el estado del modelo matemático en cualquier instante de la simulación. Esta capacidad, que se denomina interactividad, facilita la realización de experimentos sobre el modelo simulado (Dormido, 2004).

Con el fin de ilustrar el tipo de laboratorios virtuales a los que se refiere este trabajo, se muestra un ejemplo en la Figura 1: el laboratorio virtual de un sistema de cuatro depósitos, que es empleado para demostrar diferentes aspectos de la teoría de control automático multivariable (Johansson, 2000; Dormido y Esquembre, 2003). El problema de control consiste en controlar el nivel de líquido en los dos depósitos inferiores, manipulando para ello la tensión de entrada $\left(\mathrm{v}_{1}, \mathrm{v}_{2}\right)$ a las dos bombas. Cada bomba está representada en la vista del laboratorio mediante un triángulo inscrito en una circunferencia (véase la Figura 1). Los dos niveles de consigna están señalados mediante dos flechas horizontales situadas al lado de los depósitos inferiores. Obsérvese que una válvula de doble vía distribuye en dos depósitos el flujo de líquido proporcionado por cada bomba. 
Este laboratorio virtual permite experimentar con dos estrategias de control diferentes -control manual y PID descentralizado- entre las cuales el usuario puede conmutar en cualquier instante. Para control PID, el usuario puede variar los valores de los parámetros del controlador, y para control manual puede manipular directamente la tensión aplicada a las bombas $\left(\mathrm{v}_{1}, \mathrm{v}_{2}\right)$ y la proporción de flujo en cada una de las vías de las dos válvulas $\left(\mathrm{g}_{1}, \mathrm{~g}_{2}\right)$. También pueden modificarse características geométricas del sistema, tales como el área de la base y la forma de cada uno de los cuatro depósitos, y el diámetro del orificio situado en la base de cada uno de los depósitos, por el que sale el líquido. Finalmente, el usuario puede modificar en cualquier instante que desee el nivel de líquido en cada uno de los cuatro depósitos.

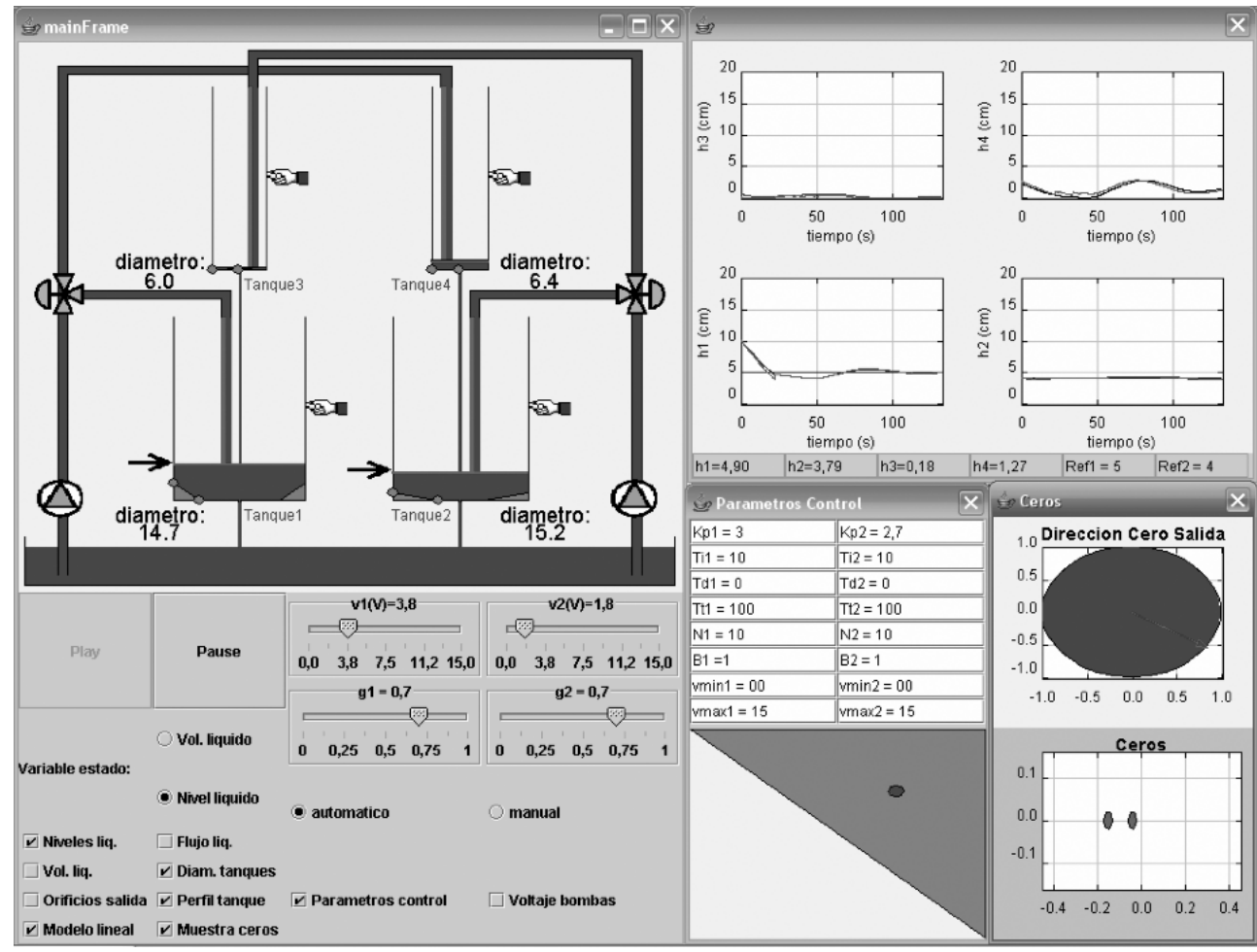

Figura 1. Laboratorio virtual del sistema de cuatro depósitos (Martín-Villalba et al., 2004)

La vista del laboratorio virtual de los cuatro depósitos está compuesta por una ventana principal (véase la parte izquierda de la Figura 1) y por ventanas secundarias con gráficas (situadas en la parte derecha). La ventana principal contiene en la 
parte superior una representación gráfica animada del sistema, que evoluciona reproduciendo la dinámica del modelo calculada mediante simulación, y también contiene, en la parte inferior, los botones de control.

Tanto la representación animada como los botones de control permiten experimentar con el modelo. Los niveles de líquido, la forma de cada depósito y los valores de consigna para los niveles de líquido pueden ser modificados pinchando y arrastrando con el ratón sobre la representación gráfica animada. Las propiedades gráficas de la representación animada están enlazadas con las respectivas variables del modelo, de modo que hay un flujo bidireccional de información.

Los deslizadores situados bajo la representación del sistema permiten modificar interactivamente los parámetros del controlador PID y el diámetro de los orificios de salida del líquido, también permiten seleccionar las variables de estado del modelo (Martín-Villalba et al., 2004), y abrir y cerrar las ventanas situadas en la parte derecha. Estas ventanas contienen gráficas de la evolución temporal de los niveles y volúmenes de líquido en los depósitos, de los flujos y de los voltajes aplicados a las bombas. Algunos de estos gráficos son mostrados en la parte derecha de la vista del laboratorio virtual, en la Figura 1.

En este artículo se presenta un curso a distancia sobre el diseño y desarrollo de laboratorios virtuales, que está dirigido a profesores de materias de Ciencias. El principal objetivo del curso es conseguir que el alumno, tras finalizar el mismo, adquiera la capacidad de diseñar y programar laboratorios virtuales útiles como herramientas docentes. De esta forma, los profesores que toman parte en el curso pueden hacer un uso eficaz de la simulación por ordenador en sus clases. El laboratorio de los cuatro depósitos sirve de referente acerca del tipo de laboratorios que se enseña a realizar en el curso: laboratorios que contienen representaciones animadas interactivas, gráficas de la evolución de ciertas variables, controles tales como deslizadores, botones, etc. Además, los laboratorios virtuales pueden incluir contenido educativo relacionado con su temática en páginas HTML.

La herramienta que se emplea en el curso, y cuyo uso se recomienda a nuestros alumnos, es la misma que se ha empleado para realizar el laboratorio de los cuatro depósitos: Easy Java Simulations, denominada comúnmente Ejs (Esquembre, 2005; Esquembre, 2008). Ejs es una herramienta informática que ha sido diseñada y programada por el Dr. D. Francisco Esquembre, profesor del Departamento de Matemáticas de la Universidad de Murcia (España). A continuación, se explican 
los motivos por los cuáles esta herramienta resulta especialmente adecuada para el desarrollo de laboratorios virtuales con aplicación docente.

\section{¿POR QUÉ EASY JAVA SIMULATIONS?}

Ejs es una herramienta software que ha sido concebida para facilitar que educadores y estudiantes, a los que se suponen escasos conocimientos de programación, desarrollen por sí mismos sus propios laboratorios virtuales (Esquembre, 2005). Ejs contiene una interfaz gráfica compuesta por una serie de paneles que van guiando al usuario en la tarea de definir el laboratorio virtual. Una vez finalizada la definición, Ejs genera automáticamente (sin intervención del usuario) el código ejecutable del laboratorio virtual.

Entre las razones por las cuales consideramos que Ejs es una herramienta adecuada para el desarrollo de laboratorios virtuales en el ámbito de la educación preuniversitaria, cabe destacar las siguientes:

- $\quad$ Ejs es muy fácil de instalar. La instalación de Ejs consiste únicamente en copiar un directorio que contiene todos los ficheros necesarios. Por otra parte, puesto que Ejs está escrito en lenguaje Java, y los laboratorios virtuales que genera Ejs están también escritos en Java, para poder ejecutar Ejs y los laboratorios generados por Ejs es necesario tener instalado el entorno de ejecución de Java.

- $\quad$ Ejs es muy fácil de usar. Ejs va guiando al usuario en el proceso de creación del laboratorio virtual, que incluye la definición de la narración, el modelo matemático y la vista.

- Ejs es gratuito y de código abierto. Esto significa que puede descargarse gratuitamente de Internet (Esquembre, 2008), y que puede ser usado y distribuido libremente. Asimismo, el entorno de ejecución de Java puede descargarse gratuitamente de Internet.

- Ejs genera automáticamente el laboratorio virtual como una aplicación Java y como páginas HTML conteniendo la simulación interactiva como un applet de Java. Esto permite que el laboratorio virtual pueda ser ejecutado como una aplicación y también que pueda ser publicado en Internet. 
Debido al hecho de que Ejs es gratuito, la UNED puede ofrecer el curso "Laboratorios virtuales interactivos: un enfoque creativo para la enseñanza de las Ciencias en el aula y a través de Internet" a un precio razonable, y una vez finalizado, los docentes que han recibido el curso pueden continuar utilizando gratis este mismo software en sus clases y distribuirlo gratuitamente a sus alumnos, para que éstos experimenten modificando laboratorios virtuales ya programados e incluso diseñen sus propios laboratorios.

\section{CURSOS OFRECIDOS POR LA UNED SOBRE DESARROLLO DE LABORATORIOS VIRTUALES}

El curso "Laboratorios virtuales interactivos: un enfoque creativo para la enseñanza de las Ciencias en el aula y a través de Internet" pertenece al Programa de Formación del Profesorado de la UNED (UNED, 2008). Se imparte a distancia y requiere aproximadamente 120 horas de trabajo del alumno. El curso, que está dirigido a profesores preuniversitarios de materias de Ciencias (física, matemáticas, química, biología, geología, etc.), tiene como objetivo que los docentes que lo reciban adquieran los conocimientos teóricos y las habilidades prácticas necesarias para diseñar y desarrollar sus propios laboratorios virtuales útiles para la enseñanza de la materia que imparten.

El material docente del curso es enviado por correo postal. Entre estos materiales se encuentra un libro de texto (Urquía y Martín-Villalba, 2007) que, partiendo de la exposición de los aspectos más básicos del diseño y la programación de la laboratorios virtuales con Ejs, va introduciendo gradualmente los conceptos mediante ejemplos, que el alumno puede reproducir por si mismo en su propio ordenador, hasta llegar a tratar conceptos avanzados cuya aplicación permite realizar laboratorios virtuales muy sofisticados. En la Sección 4 se describirá brevemente el contenido del curso. Finalmente, con el fin de mostrar hasta qué punto el curso cumple con su objetivo, en la Sección 5 se presentan algunos de los laboratorios desarrollados por los alumnos.

Por otra parte, en el sitio web http://www.euclides.dia.uned.es/simulab-pfp se ofrece gratuitamente una versión online reducida y escrita en lengua inglesa del curso del Programa de Formación del Profesorado. Desde este sitio web, puede también accederse a los laboratorios virtuales explicados en el curso, y a una selección de los laboratorios diseñados y programados por los alumnos. Este curso online gratuito, titulado "Virtual-lab implementation with Ejs", fue galardonado con el segundo premio en el concurso internacional "Best Designed Course Award", celebrado con 
motivo de la $6^{\text {th }}$ International Conference on Virtual University, que tuvo lugar en Bratislava (Eslovaquia) en diciembre de 2005. La contribución presentada a dicha conferencia es "A Distance Learning Course on Virtual-lab Implementation for High School Science Teachers" (Martín-Villalba et al., 2005).

\section{CONTENIDO DE LOS CURSOS}

El curso "Laboratorios virtuales interactivos: un enfoque creativo para la enseñanza de las Ciencias en el aula y a través de Internet" se encuentra estructurado en las tres partes siguientes (se muestra entre paréntesis una estimación del esfuerzo que debe dedicar el alumno a cada parte):

- Parte I. Fundamentos del modelado y la simulación (10\%)

- $\quad$ Parte II. Easy Java Simulations (30\%)

- $\quad$ Parte III. Casos de estudio (6o\%)

A continuación, se describe brevemente el contenido de cada una de las tres partes. El contenido del curso se muestra en la Tabla 1. El desarrollo en detalle de cada una de las partes puede encontrarse en el texto "Diseño, programación y distribución a través de Internet de laboratorios virtuales empleando Easy Java Simulations" (Urquía y Martín-Villalba, 2007).

Por otra parte, el curso online gratuito "Virtual-lab implementation with Ejs" consta únicamente de siete lecciones. Las seis primeras coinciden con las mostradas en la Tabla 1. La Lección 7 del curso online engloba las nueve lecciones de la Parte III, es decir, contiene los nueve casos de estudio, incluyendo la posibilidad de descargar el código fuente de los nueve laboratorios virtuales.

\section{Parte I. Fundamentos del modelado y la simulación}

Lección 1. Conceptos básicos del modelado y la simulación

Lección 2. Simulación de modelos de tiempo continuo

\section{Parte II. Easy Java Simulations}

Lección 3. Fundamentos de Ejs

Lección 4. Instalación y arranque de Ejs

Lección 5. Conceptos básicos para la descripción del modelo

Lección 6. Conceptos básicos para la descripción de la vista 


\section{Parte III. Casos de estudio}

Lección 7. Programación de un osciloscopio virtual con Ejs

Lección 8. Laboratorio virtual del concepto de ciclo límite

Lección 9. Principio de Arquímedes

Lección 10. Péndulo simple

Lección 11. Conducción de calor a través de una pared múltiple

Lección 12. Laboratorio virtual de un sistema mecánico

Lección 13. Cálculo del número pi por el método de Monte Carlo

Lección 14. Simulación interactiva de un globo aerostático

Lección 15. Laboratorio virtual del sistema bola y varilla

Tabla 1. Contenido del curso "Laboratorios virtuales interactivos: un enfoque creativo para la enseñanza de las Ciencias en el aula y a través de Internet"

\section{Fundamentos del modelado y la simulación}

En la Lección 1 se explica el significado de conceptos fundamentales, tales como "sistema", "modelo", "experimento" y "simulación". Se describen los diferentes tipos de modelos: verbales, mentales, físicos y matemáticos. Se discuten diferentes clasificaciones de los modelos matemáticos (Ljung y Glad, 1994), incluyendo las siguientes: deterministas vs. estocásticos; dinámicos vs. estáticos; y de tiempo continuo vs. de tiempo discreto vs. híbridos. Finalmente, se explica el concepto de "marco experimental de un modelo" (Cellier, 1991).

La Lección 2 está dedicada a la simulación por ordenador de modelos de tiempo continuo. Se introducen los conceptos de "ecuación" y "variable", y se establece la clasificación de las variables del modelo en parámetros, variables algebraicas y variables de estado (Cellier, 1991; Elmqvist, 1978). Se describe un algoritmo para la simulación por ordenador de modelos de tiempo continuo, y se ilustra su aplicación mediante varios ejemplos. Finalmente, se explica un método sistemático para realizar la asignación de la causalidad computacional de cualquier modelo de tiempo continuo (Elmqvist, 1978), y se aplica este algoritmo en varios ejemplos.

La Lección 2 proporciona la base para comprender el algoritmo de la simulación de Ejs, así como las manipulaciones simbólicas que debe realizar el usuario de Ejs sobre el modelo matemático del laboratorio virtual, a fin de formularlo de manera 
que pueda ser simulado empleando Ejs. Estos dos temas son tratados en profundidad en la Lección 5 .

\section{Easy Java Simulations}

Las Lecciones 3 y 4 constituyen una introducción a Ejs, en la que se explica su base conceptual: una simplificación original del paradigma modelo-vista-control (Esquembre, 2004). También se explica, con todo detalle, cómo instalar Ejs y cómo ejecutar y publicar en Internet un laboratorio virtual que ya se encuentre programado. La Lección 5 describe el procedimiento que debe emplear el usuario de Ejs para la definición del modelo del laboratorio virtual. Este procedimiento está íntimamente relacionado con el algoritmo de la simulación de Ejs, que es el algoritmo para la simulación de modelos de tiempo continuo (descrito en la Lección 2) modificado de modo que permita la interactividad. La Lección 6 es una introducción a la definición de la vista del laboratorio virtual usando Ejs. Se describen algunos de los elementos gráficos interactivos más comúnmente empleados, entre los que proporciona Ejs. El resto de los elementos de Ejs para la composición de la vista del laboratorio virtual y el procedimiento para incluir el contenido docente del laboratorio virtual se explican en los ejemplos de la Parte III.

\section{Casos de estudio}

La tercera parte del curso es un tutorial sobre el diseño y programación de laboratorios virtuales usando Ejs. Consiste en una sucesión de casos de estudio de complejidad creciente. Se aplican los conceptos expuestos en las Partes I y II, y se introducen nuevos conceptos más avanzados. Se supone que los estudiantes deben seguir las Lecciones 7 a 15 sentados delante de su ordenador, programando ellos mismos los laboratorios virtuales. Además de las explicaciones, se proporciona al alumno el código fuente del laboratorio correctamente programado y documentado, con lo cual el alumno podrá resolver por sí mismo la mayoría de las cuestiones que le surjan. Esta parte del curso supone aproximadamente el 60\% del esfuerzo del alumno, con lo cual puede considerarse que éste es un curso de carácter eminentemente práctico.

Los modelos matemáticos de estos laboratorios virtuales (véase la Tabla 1) cubren la mayoría de los tipos de modelos descritos en la Lección 1. Los modelos del "principio de Arquímedes" y de la "conducción de calor a través de una pared múltiple" son estáticos. Por el contrario, los modelos de "el osciloscopio virtual", 
"el ciclo límite”, "el péndulo simple”, "el sistema mecánico” y "el sistema de bola y varilla” son dinámicos.

Los ejemplos han sido escogidos de modo que cubran los diferentes tipos de modelos matemáticos: (1) el modelo de "el osciloscopio virtual" está únicamente compuesto por ecuaciones algebraicas; (2) el modelo de "el ciclo límite” está compuesto únicamente por ecuaciones diferenciales ordinarias; (3) el modelo de "el péndulo simple" y de "el globo aerostático" está compuesto de ecuaciones algebraicas y diferenciales (DAE); (4) el modelo de "el sistema de bola y varilla" es híbrido; y (5) el modelo de "el sistema mecánico" es DAE-híbrido con estructura variable. Finalmente, el modelo del laboratorio para "la estimación del valor del número pi" aplica una técnica estocástica: el método de Monte Carlo.

En las Figuras 2 a 5 se muestran cuatro de estos laboratorios virtuales. El laboratorio virtual de la pared multicapa se emplea para ilustrar la conducción de calor, en el estado estacionario, a través de una pared compuesta por tres capas de materiales diferentes (véase la Figura 2). El usuario del laboratorio puede modificar el espesor (casillas señaladas como $\mathrm{L}_{\mathrm{a}}, \mathrm{L}_{\mathrm{b}}$ y $\mathrm{L}_{\mathrm{c}}$ ) y la conductividad térmica (casillas señaladas como $\mathrm{k}_{\mathrm{a}}, \mathrm{k}_{\mathrm{b}}$ y $\mathrm{k}_{\mathrm{c}}$ ) de cada una de las tres capas, así como las temperaturas exterior (casilla $\mathrm{T}_{\mathrm{ext}}$ ) e interior (casilla $\mathrm{T}_{\mathrm{int}}$ ). Para cada nueva condición introducida por el usuario, el laboratorio calcula y muestra el perfil de temperatura en la pared (véase la gráfica en la parte derecha de la Figura 2).

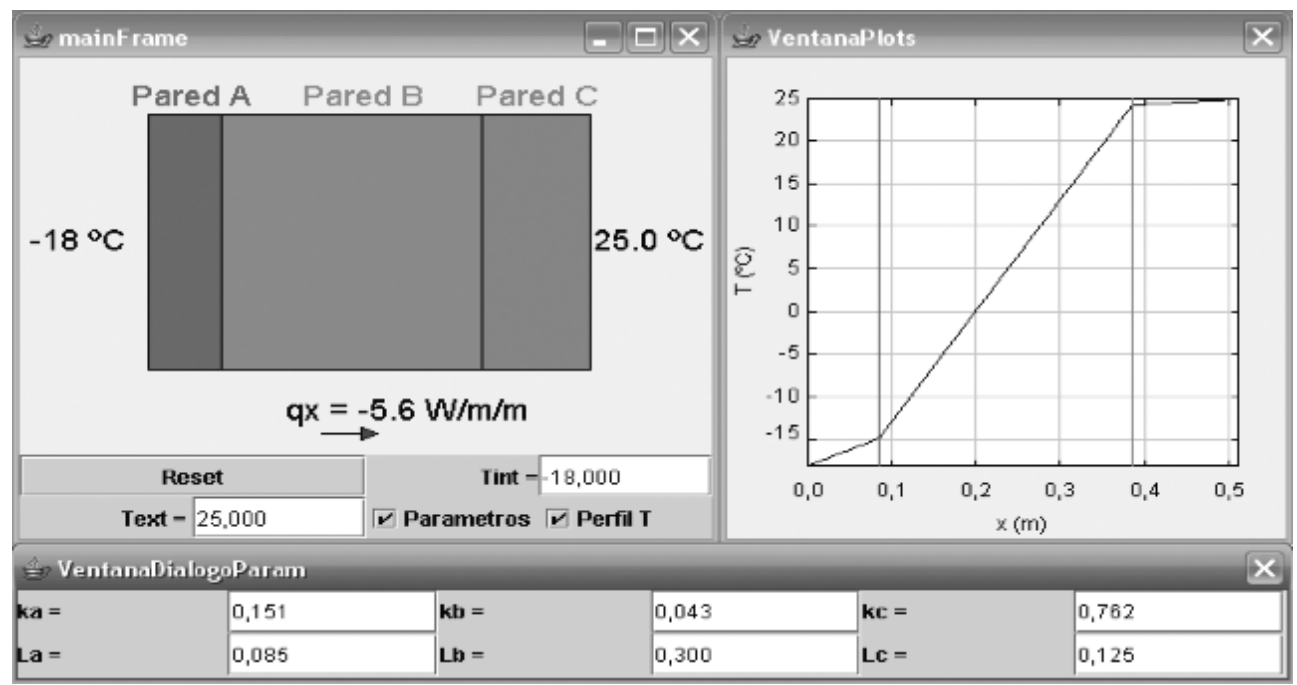

Figura 2. Laboratorio virtual de una pared con tres capas 


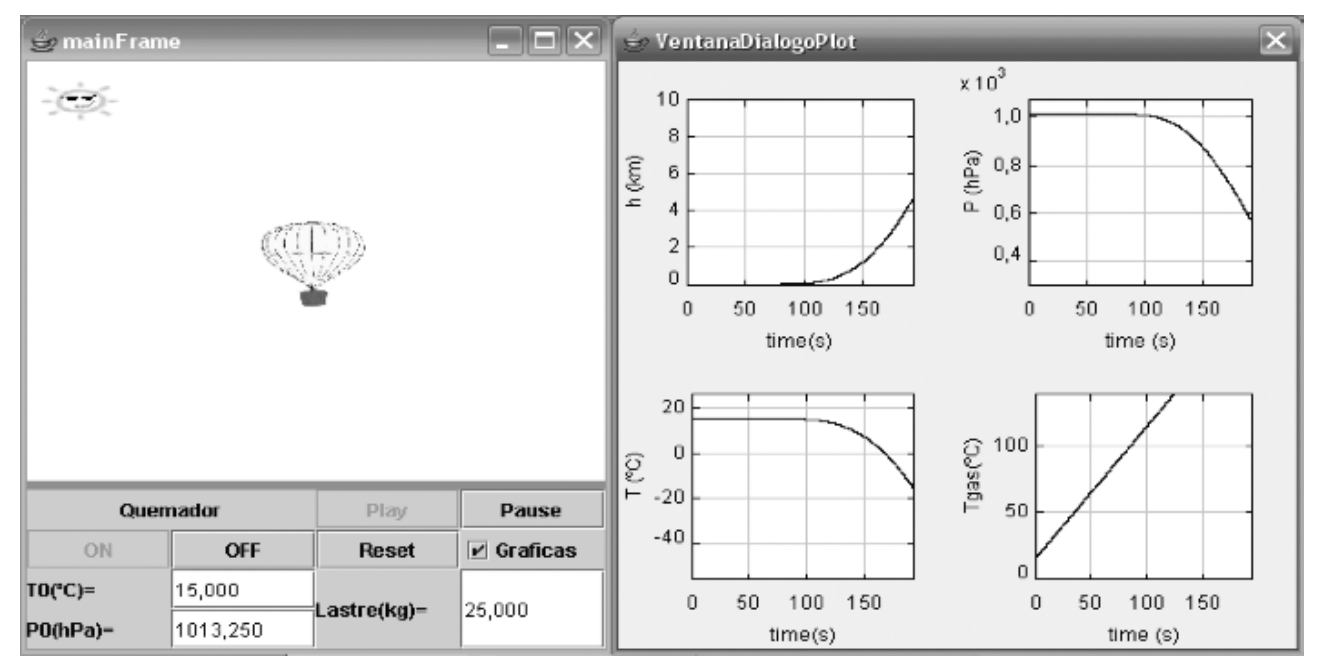

Figura 3. Laboratorio virtual de un globo aerostático

El laboratorio virtual mostrado en la Figura 3 emula el comportamiento de un globo aerostático. El usuario puede modificar la temperatura y la presión atmosférica, el peso empleado como lastre, y también puede encender y apagar el quemador. Estos cuatro factores influyen en la dinámica del globo, que es calculada mediante la simulación del modelo matemático por Ejs, y es representada mediante el movimiento vertical del dibujo del globo (mostrado en la ventana izquierda de la Figura 3) y mediante la gráfica de la evolución temporal de la altura (mostrada en la parte derecha de la Figura 3). También, se grafica la evolución temporal de la presión y la temperatura atmosférica (manipuladas directamente por el usuario del laboratorio), y de la temperatura del gas contenido en el interior del globo (controlada manipulando el encendido/apagado del quemador). 


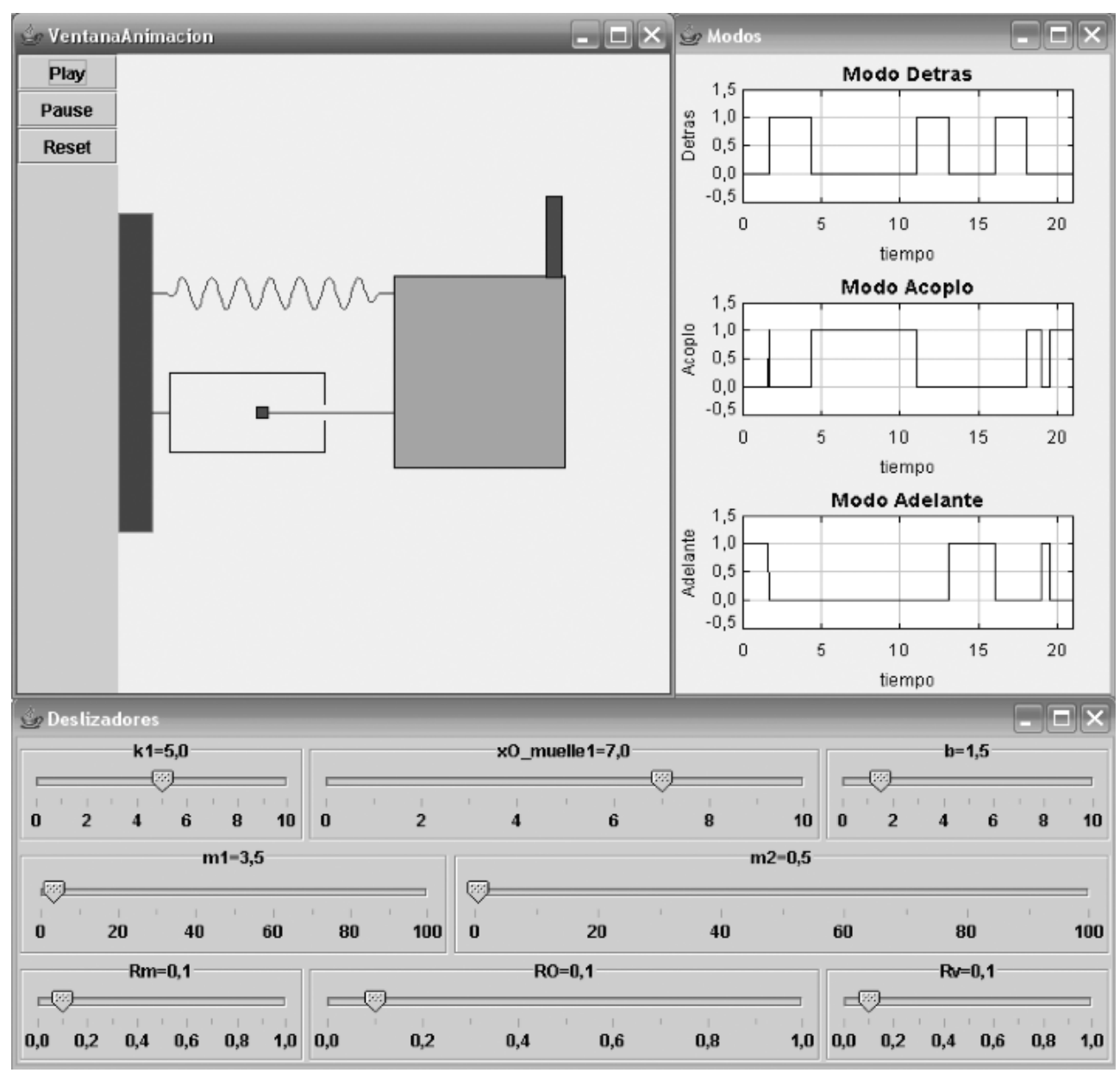

Figura 4. Laboratorio virtual de un sistema mecánico

El laboratorio virtual mostrado en la Figura 4 reproduce la dinámica de un sistema mecánico compuesto por un muelle y un amortiguador que se encuentran unidos por uno de sus extremos a una pared en reposo, y por el otro extremo a un objeto con masa que puede desplazarse. Encima de este objeto, se encuentra situado otro objeto con masa, y en la superficie de contacto entre ambos existe fricción. Los deslizadores situados en la parte inferior del laboratorio virtual permiten modificar los parámetros de las relaciones constitutivas del muelle y del amortiguador, las masas de los dos objetos y los parámetros del modelo de la fricción, que se supone Coulombiana. 
La parte superior del laboratorio virtual consta de tres ventanas. La ventana situada a la izquierda contiene tres botones (Play, Pause y Reset), que permiten controlar la ejecución de la simulación: arrancarla, detenerla y reiniciarla asignando ciertos valores por defecto al estado del sistema. La ventana central contiene una representación esquemática animada del sistema, que reproduce su comportamiento dinámico. Si se detiene la simulación, puede modificarse la posición de cualquiera de las masas pinchando sobre ella y arrastrando con el ratón. Una vez fijadas las nuevas posiciones, pulsando el botón Play continuará la simulación partiendo de estos valores de la posición fijados interactivamente por el usuario. En la ventana de la derecha, se representa gráficamente la evolución de determinadas magnitudes relevantes del sistema.

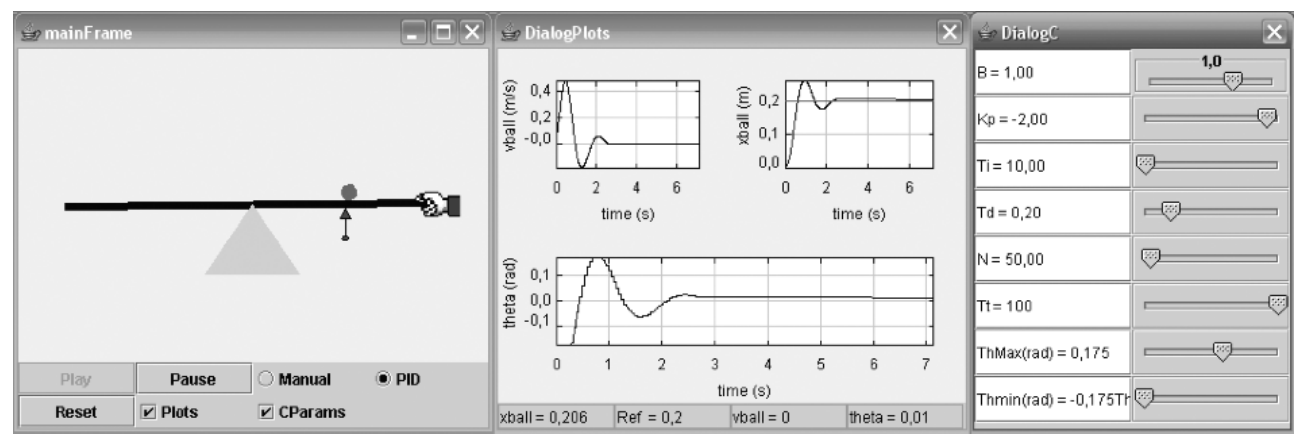

Figura 5. Laboratorio virtual del sistema "bola y varilla"

Finalmente, en la Figura 5 se muestra el laboratorio virtual del sistema "bola y varilla”. La bola rueda sobre la superficie de la varilla. El objetivo es conseguir que la posición de la bola sea una determinada (señalada mediante una flecha en la Figura 5), para lo cual hay que variar el ángulo de inclinación de la varilla. El laboratorio permite controlar este sistema manualmente y usando un controlador PID. El control manual se realiza pinchando con el ratón sobre la mano que aparece dibujada en el extremo de la varilla y arrastrando hasta la posición deseada. El valor de consigna para la posición de la bola también puede cambiarse pinchando con el ratón sobre la fecha y arrastrándola a la posición deseada. En la parte central, se muestra la evolución temporal de la velocidad y posición de la bola, y del ángulo de inclinación de la varilla. En la parte derecha del laboratorio, hay diferentes deslizadores que permiten modificar interactivamente determinadas magnitudes relevantes del sistema, tales como los parámetros del controlador PID, los valores máximo y mínimo de la inclinación de la varilla, etc. 


\section{ALGUNOS LABORATORIOS DESARROLLADOS POR LOS ALUMNOS}

La evaluación de las habilidades adquiridas (con la calificación: "apto" o "no apto") en el curso "Laboratorios virtuales interactivos: un enfoque creativo para la enseñanza de las Ciencias en el aula y a través de Internet" se basa en un trabajo que debe realizar el alumno individualmente. Este trabajo consistente en el diseño y programación, usando Ejs, de dos laboratorios virtuales de su elección: uno sencillo y otro con un nivel mayor de complejidad. Ambos laboratorios han de estar documentados, usando para ello las facilidades de Ejs. El estudiante debe desarrollar estos dos laboratorios virtuales en su propio ordenador y enviarlos al equipo docente por correo electrónico. Los conceptos ilustrados en los laboratorios virtuales quedan a elección del alumno, y típicamente están relacionados con su actividad docente. La documentación del laboratorio virtual debe incluir el siguiente contenido: (1) objetivo docente del laboratorio; (2) explicación detallada del modelo matemático y del algoritmo de la simulación; y (3) un conjunto de actividades que se proponen al alumno, a realizar con el laboratorio virtual.

Se valora tanto el correcto funcionamiento de los laboratorios virtuales como su utilidad como herramienta docente. En el sitio Web del curso puede encontrarse una selección de algunos laboratorios virtuales desarrollados por los alumnos. En la Figura 6 se muestra la página Web de acceso a dichos laboratorios. Con ello, se pretende proporcionar una idea de las habilidades adquiridas por los alumnos al realizar el curso. Algunos de estos laboratorios son descritos brevemente a continuación.

\section{Espectro de emisión del átomo de hidrógeno}

Este laboratorio ilustra cómo se produce el espectro de emisión del átomo de hidrógeno (véase la Figura 7), de acuerdo con el modelo de Bohr. El laboratorio virtual permite seleccionar los niveles de energía inicial y final de la transición, escribiéndolos en las casillas señaladas como $\mathrm{n}_{2} \mathrm{y}_{1}$ respectivamente (véase la parte superior izquierda de la Figura 7). Una vez que ha seleccionado los dos niveles de energía, el estudiante deberá pulsar el botón Play. Entonces el laboratorio muestra gráficamente el cambio en el nivel de energía del electrón (en la parte derecha del laboratorio) y dibuja la raya del espectro de emisión (en la parte inferior del laboratorio). En la Figura 7, se representa esquemáticamente la transición del electrón desde el nivel $\mathrm{n}_{2}=3$ hasta el nivel $\mathrm{n}_{1}=2$, y el espectro de emisión correspondiente. 
Educación a Distancia del Profesorado de Ciencias en el Desarrollo de Laboratorios Virtuales

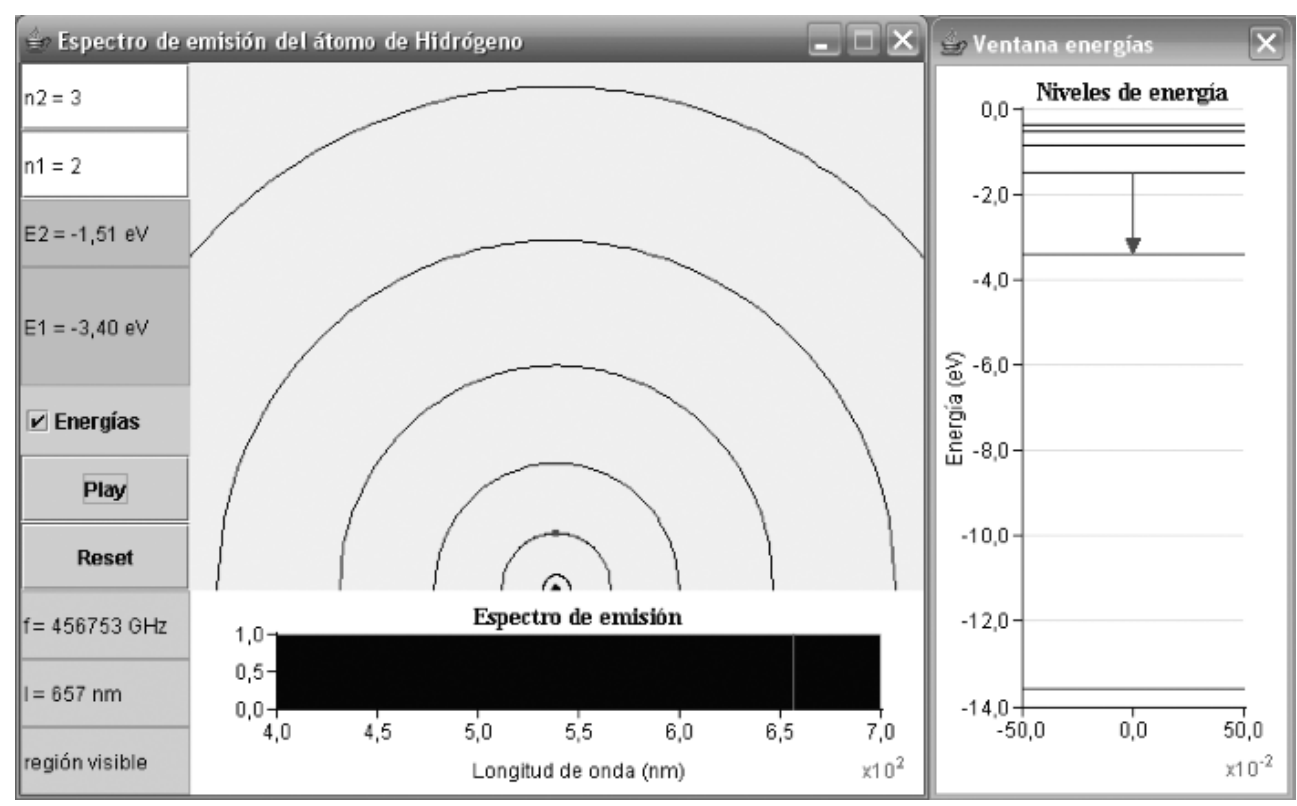

Figura 6. Una selección de los laboratorios desarrollados por los alumnos

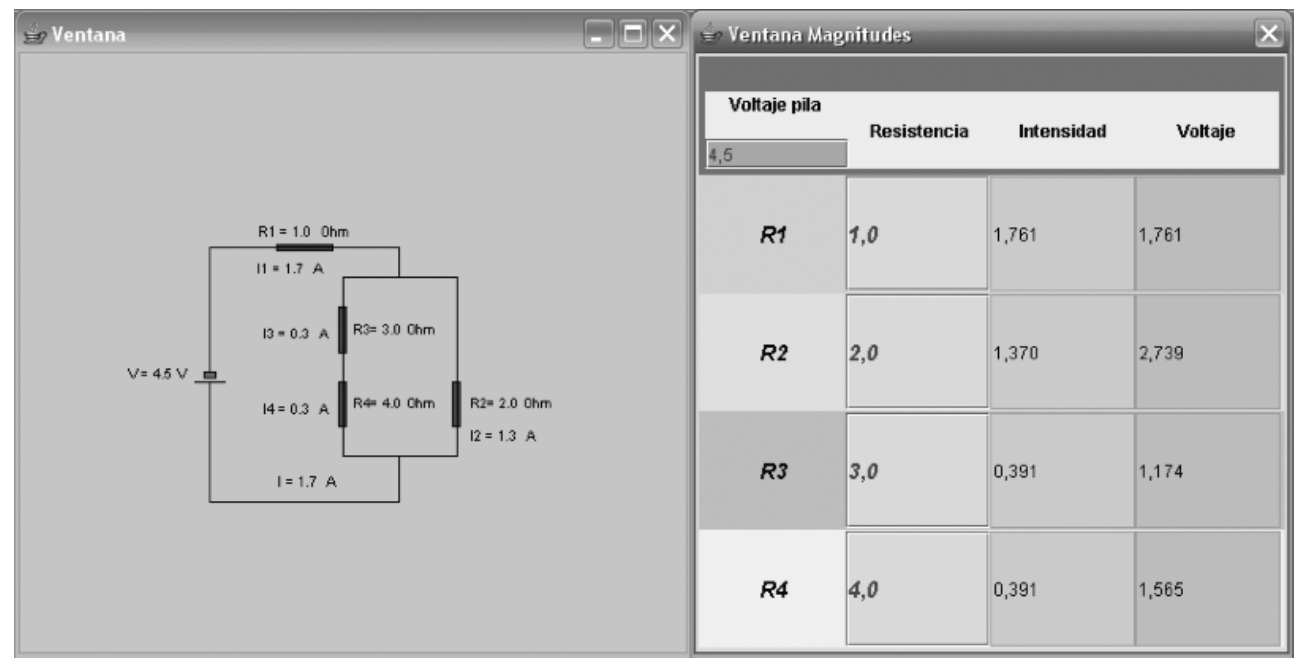

Figura 7. Laboratorio virtual del espectro de emisión del átomo de hidrógeno 


\section{Circuito eléctrico}

En la Figura 8 se muestra un laboratorio virtual útil para ilustrar conceptos básicos de la teoría de circuitos eléctricos, tales como la Ley de Ohm, y las leyes de Kirchhoff de los nudos y las mallas. El estudiante puede modificar la tensión de la batería y la magnitud de cada una de las cuatro resistencias, escribiendo los correspondientes valores en las casillas situadas en la parte derecha del laboratorio. Cada vez que el estudiante modifica uno de estos valores, el laboratorio virtual recalcula la caída de tensión en cada componente y la corriente eléctrica que circula a su través. Estos valores calculados se muestran en las casillas de la parte derecha del laboratorio y también en el esquemático del circuito representado en la parte izquierda del laboratorio (véase la Figura 8).

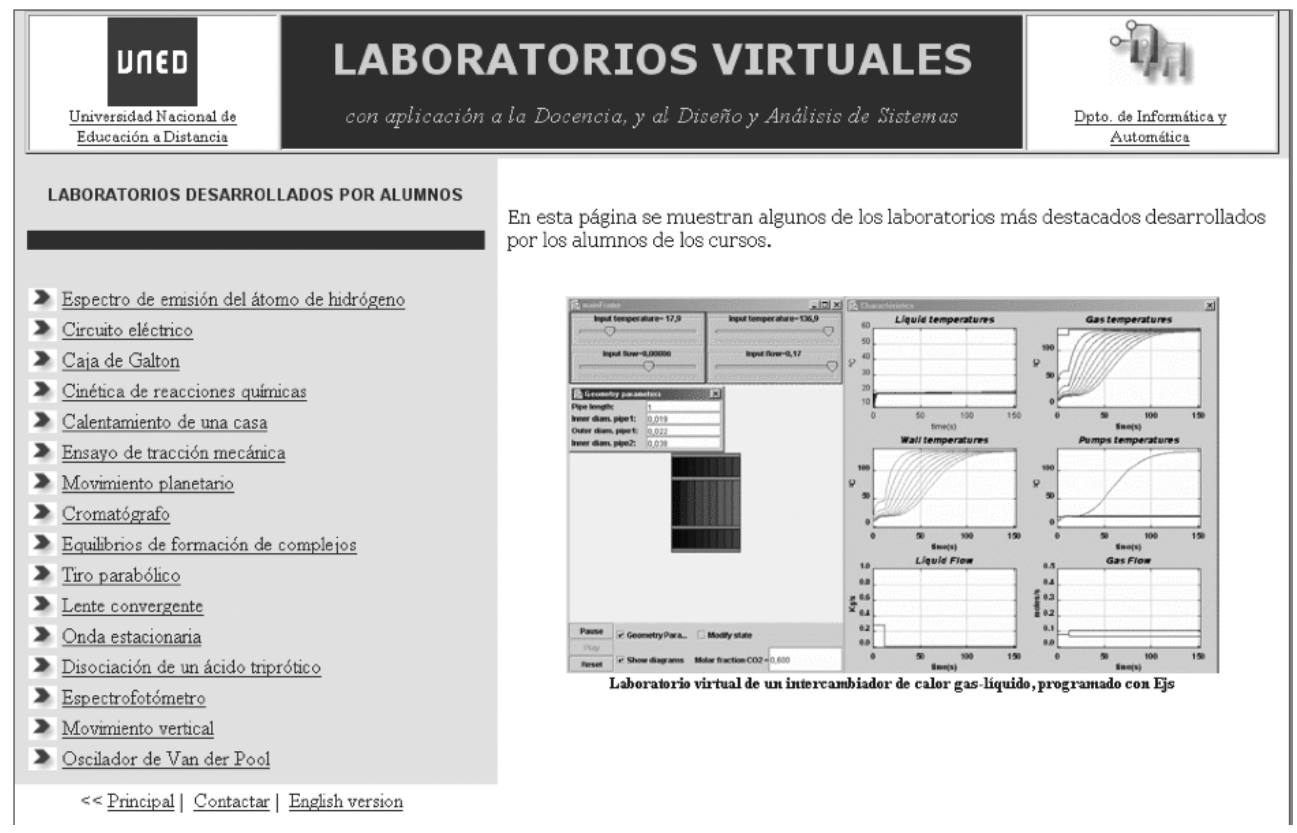

Figura 8. Laboratorio virtual de un circuito eléctrico

\section{Tablero de Galton}

El laboratorio virtual mostrado en la Figura 9 reproduce el comportamiento dinámico de un tablero de Galton, también llamado "quincunx", que es un dispositivo empleado para ilustrar el concepto de distribución de probabilidad binomial. El 
"quincunx" consiste en un tablero triangular en el cual se colocan varias filas de pivotes equiespaciados y unas cajas en la parte inferior, tal como se muestra en el diagrama de la parte izquierda del laboratorio de la Figura 9. Se van lanzando bolas, una tras otra, desde la parte superior del tablero, que van rebotando en los pivotes y finalmente quedan almacenadas en las cajas situadas en la parte inferior del tablero. Cuando una bola rebota con un pivote, tiene igual probabilidad de hacerlo hacia la derecha que hacia la izquierda.

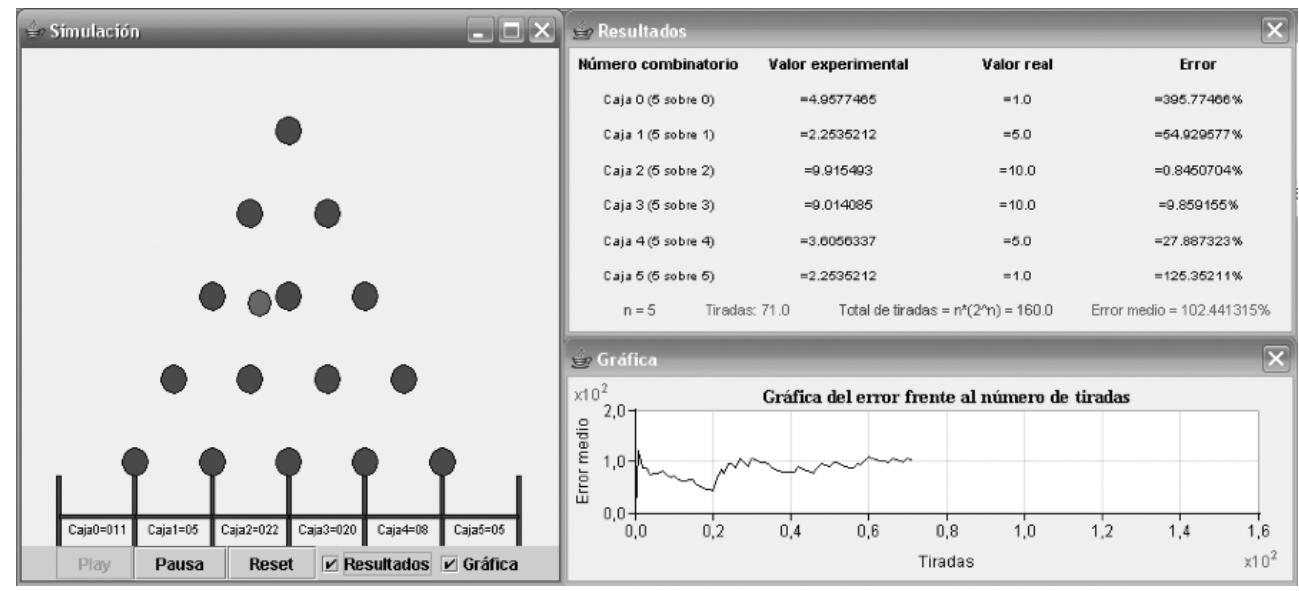

Figura 9. Laboratorio virtual del tablero de Galton

El laboratorio permite al estudiante modificar el número de cajas (y consecuentemente el de pivotes, ya que están relacionados) y el número de lanzamientos. El laboratorio virtual simula y representa gráficamente la trayectoria de las bolas en el tablero, y va indicando el número de bolas que caen en cada caja, así como de la diferencia entre este número y el predicho por una distribución de probabilidad binomial. Estos cálculos, así como la evolución del error con el número de lanzamientos, se muestran en la parte derecha del laboratorio.

Obsérvese que los dos laboratorios anteriores contenían modelos matemáticos estáticos. Cuando el estudiante modifica las condiciones experimentales, el laboratorio calcula y muestra gráficamente el estado del modelo correspondiente a estas nuevas condiciones iniciales, y queda a la espera, sin realizar nuevos cálculos, hasta que el estudiante modifique nuevamente las condiciones experimentales. Por el contrario, el laboratorio del tablero de Galton calcula y representa gráficamente el comportamiento dinámico de las bolas, es decir, la evolución temporal del estado del modelo. Se trata de un modelo dinámico. 


\section{Cinética de reacciones químicas}

El laboratorio mostrado en la Figura 10 se emplea para simular la evolución temporal de las especies involucradas en las reacciones químicas siguientes:
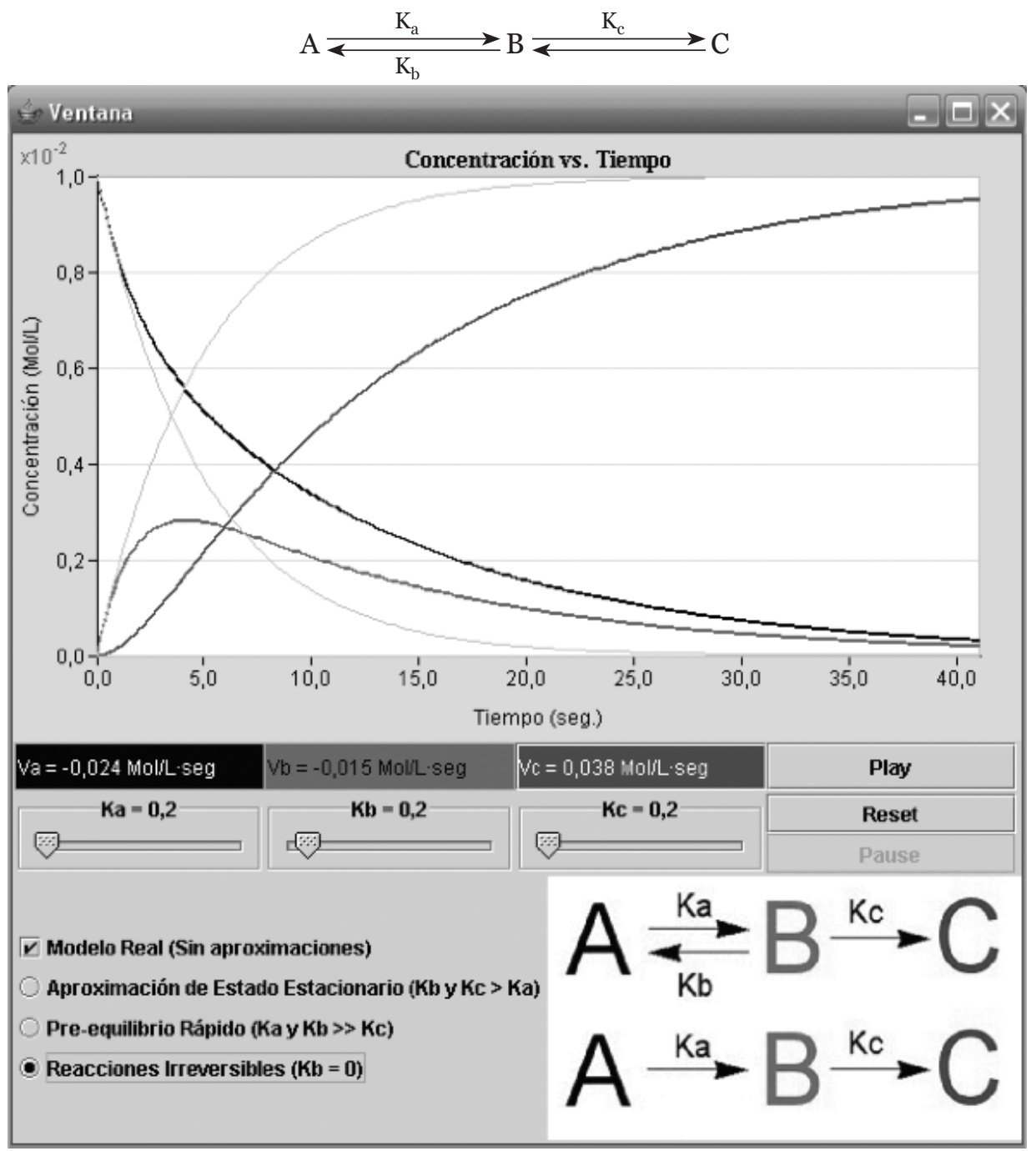

Figura 10. Laboratorio virtual de una reacción química

Pueden modificarse los coeficientes de la velocidad de reacción $\left(\mathrm{K}_{\mathrm{a}}, \mathrm{K}_{\mathrm{b}} \mathrm{y} \mathrm{K}_{\mathrm{c}}\right)$, empleando los tres deslizadores situados en la mitad inferior del laboratorio. 
Asimismo, el laboratorio permite al estudiante escoger la hipótesis a emplear para la realización de los cálculos de la cinética: estado estacionario, rápido pre-equilibrio y reacciones irreversibles. Para las condiciones seleccionadas, el laboratorio virtual calcula y representa gráficamente la evolución de la concentración de cada una de las especies (véase la gráfica en la parte superior del laboratorio).

\section{Balance energético de una vivienda}

Finalmente, en la Figura 11 se muestra un laboratorio virtual diseñado para calcular y representar gráficamente la influencia de diferentes factores en el balance energético en una vivienda, tales como el flujo de calor a través de las paredes, las puertas, las ventanas, el techo y el suelo. El laboratorio permite que el estudiante seleccione: (1) los materiales y espesores de las paredes y del techo; (2) la eficiencia térmica de las ventanas, las puertas y el suelo; y (3) la temperatura en el interior de la casa y en el exterior.

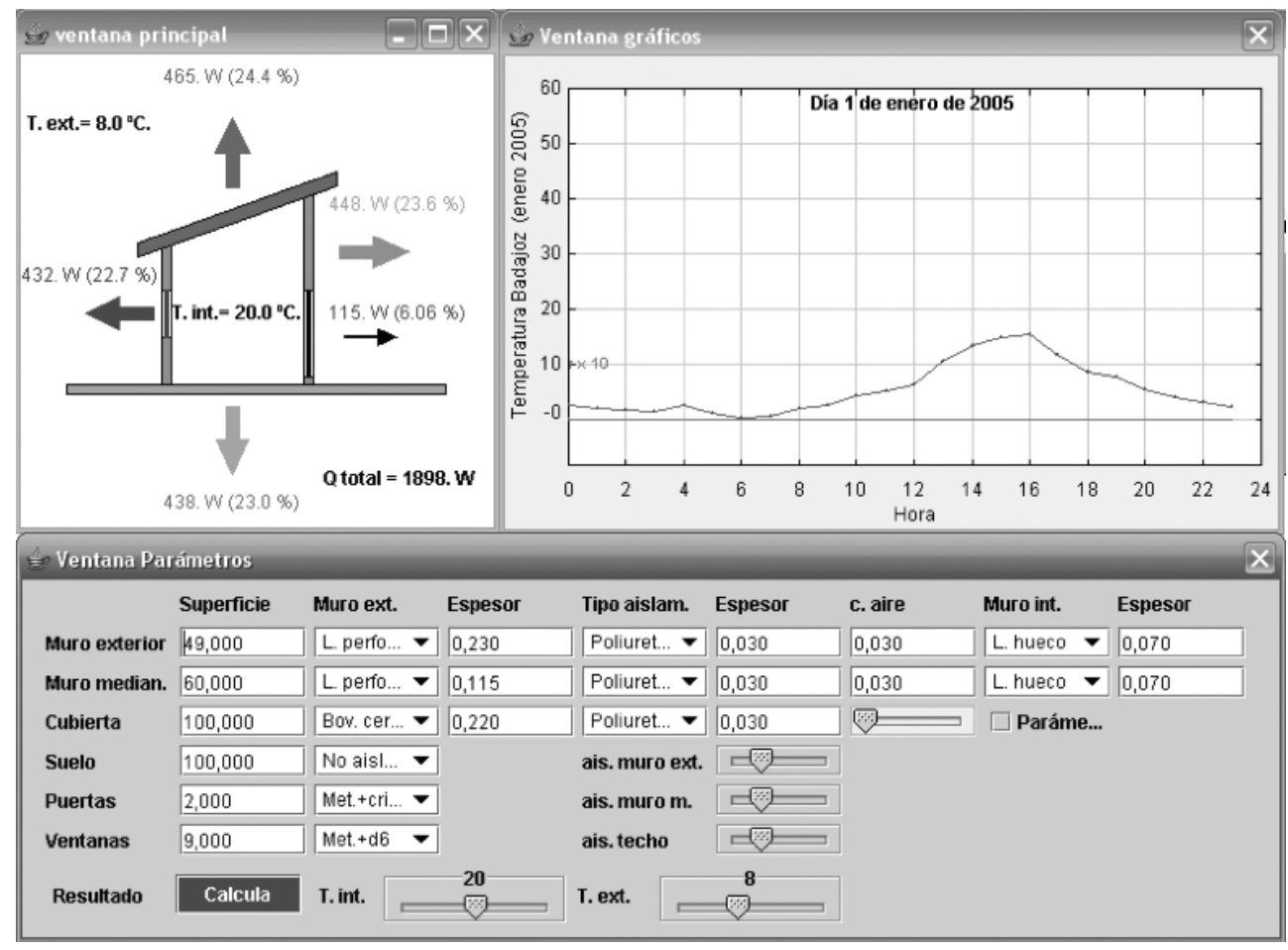

Figura 11. Laboratorio virtual del balance energético de una vivienda 
Además, el laboratorio calcula el coste diarioy mensual de cuatro tipos alternativos de sistema de calefacción: gasoil, hilo radiante, bomba de calor y eléctrico. Las temperaturas exteriores empleadas para realizar estos cálculos son las registradas durante el mes de enero de 2005, en la ciudad española de Badajoz.

\section{CONCLUSIONES}

Tras discutir la utilidad de los laboratorios virtuales como herramientas docentes, se han presentado dos cursos ofrecidos por la UNED, dirigidos a profesores preuniversitarios de materias de Ciencias, cuyo objetivo es que estos profesores adquieran la capacidad de diseñar y desarrollar laboratorios virtuales útiles para la docencia de las materias que imparten. Se han fundamentado los motivos que han llevado a elegir Ejs como herramienta informática para la programación de los laboratorios virtuales.

El primero de los cursos ofrecidos por la UNED, "Laboratorios virtuales interactivos: un enfoque creativo para la enseñanza de las Ciencias en el aula y a través de Internet", con una dedicación aproximada de 120 horas, forma parte del Programa de Formación del Profesorado de la UNED. Se ha discutido el diseño, estructura y contenido del curso. También se ha descrito el criterio de evaluación empleado. Finalmente, se han mostrado varios laboratorios virtuales realizados por los alumnos de este curso. A la vista de estos laboratorios virtuales, puede afirmarse que el curso cumple los objetivos planteados.

El segundo curso, "Virtual-lab implementation with Ejs", es una versión resumida del anterior. Se trata de un curso gratuito, basado en web, que está escrito en lengua inglesa. El sitio web de este curso online gratuito es: http://www.euclides.dia.uned. es/simulab-pfp

\section{Agradecimientos}

Nos gustaría mostrar nuestro agradecimiento a los alumnos de la promoción 2005 de nuestro curso del Programa de Formación del Profesorado de la UNED, que amablemente nos han autorizado a publicar sus laboratorios virtuales en el sitio web del curso, y en particular a los autores de los laboratorios virtuales mostrados en este trabajo: D. Jorge Martín Ortiz (espectro de emisión del átomo de hidrógeno), D. Francisco Silva Rodríguez (circuito eléctrico), D. Juan de la Cruz Hidalgo (tablero de 
Galton), D. Emilio Bustelo Gutiérrez (cinética de reacciones químicas) y D. Manuel Bueno Santos (balance energético de una vivienda).

\section{REFERENCIAS BIBLIOGRÁFICAS}

Burbeck, S. (1997). Applications Programming in Smalltalk-8o(TM): How to use Model-View-Controller $(M V C)$. [en línea] Archivo UIUC Smalltalk Archive. Disponible en: http:// st-www.cs.uiuc.edu/users/smarch/stdocs/mvc.html [consulta 2007, 9 de mayo].

Cellier, F. E. (1991). Continuous System Modeling. Springer-Verlag.

Dormido, S. (2004). Control learning: present and Future. Annual Reviews in Control, 28, 115-136.

Dormido, S.; Esquembre, F. (2003). The Quadruple-Tank Process: An Interactive Tool for Control Education, en: proceedings de 2003 European Control Conference. Cambridge: Reino Unido.

Elmqvist, H. (1978). A Structured Model Language for Large Continuous Systems. Tesis Doctoral. Suecia: Lund Institute of Technology.

Esquembre, F. (2004). Easy Java Simulations: a Software Tool to Create Scientific Simulations in Java. Computer Physics Communications, 156, 199-204.

Esquembre, F. (2005). Creación de Simulaciones Interactivas en Java. Pearson Prentice-Hall.

Jimoyiannis, A.; Komis, V. (2001). Computer simulations in physics teaching and learning. Computers \& Education, 36, 183-204.

Johansson, K. H. (2000). The QuadrupleTank Process: A Multivariable Laboratory Process with an Adjustable Zero. IEEE Transactions on Control Systems Technology, Vol. 8, No. 3 (May), 456465.
Ljung, L.; Glad, T. (1994). Modeling of Dynamic Systems. New Jersey: Prentice Hall.

Martín-Villalba, C.; Muñoz, R.; Urquía, A.; Dormido, S. (2005). A Distance Learning Course on Virtual-lab Implementation for High School Science Teachers, en: proceedings de $6^{\text {th }}$ International Conference Virtual University, Bratislava, Eslovaquia, 3-8.

Martín-Villalba, C.; Urquía, A.; Sánchez, J.; Dormido, S.; Esquembre, F.; Guzmán, J. L.; Berenguel, M. (2004). Interactive simulation of object-oriented hybrid models, by combined use of Ejs, Matlab/ Simulink and Modelica/Dymola, en: proceedings de $18^{\text {th }}$ EuropeanSimulation Multiconference, Magdeburgo, Alemania, 210-215.

Urquía, A.; Martín-Villalba, C. (2007). Diseño, programación y distribución a través de Internet de laboratorios virtuales empleando Easy Java Simulations. Texto base del curso "Laboratorios virtuales interactivos: un enfoque creativo para la enseñanza de las Ciencias en el aula y a través de Internet" del Programa de Formación del Profesorado de la UNED. 


\section{PERFIL ACADÉMICO Y PROFESIONAL DE LOS AUTORES}

Carla Martin Villalba obtuvo el título de Ingeniero en Electrónica por la Universidad Complutense de Madrid (España) en el año 2001 y el título de Doctor en Informática en el año 2007 por la UNED (España). Desde el año 2005, ocupa el puesto de Profesor Ayudante en el Dpto. de Informática y Automática de la UNED.

E-mail: carla@dia.uned.es

Alfonso Urquía Moraleda obtuvo el título de Licenciado en CC. Físicas en 1992 por la Universidad Complutense de Madrid (España) y el título de Doctor en CC. Físicas en 2000 por la UNED (España). Su experiencia laboral incluye 6 años como ingeniero de I+D en AT\&T y Lucent Technologies - Bell Labs. Desde el año 1995 ha ocupado diferentes puestos de profesor en el Dpto. de Informática y Automática de la UNED, donde desde 2003 es Profesor Titular de Universidad en el área "Ingeniería de Sistemas y Automática".

E-mail: aurquia@dia.uned.es

Sebastián Dormido Bencomo obtuvo el título de Licenciado en CC. Físicas por la Universidad Complutense de Madrid (1968) y el título de Doctor por la Universidad del País Vasco (1971). En 1981 fue nombrado Catedrático de Ingeniería de Sistemas y Automática en la UNED. Ha supervisado más de 25 Tesis Doctorales y es coautor de más de 100 artículos en revistas internacionales. Entre los años 2002 y 2007 ha ocupado el puesto de Presidente de CEA-IFAC.

E-mail: sdormido@dia.uned.es

DIRECCIÓN DE LOS AUTORES:

Departamento de Informática y Automática UNED

Juan del Rosal 16, 28040 Madrid, España

Fecha de recepción del artículo: 29/05/07

Fecha de aceptación del artículo: 29/01/08 